\title{
Aircraft position estimation using angle of arrival of received radar signals
}

\author{
Freeha Majeed Amjad, Ahmad Zuri Sha'ameri, Kamaludin Mohamad Yusof, Paulson Eberechi \\ School of Electrical Engineering, Faculty of Engineering, Universiti Teknologi Malaysia, Malaysia
}

\begin{tabular}{|c|c|}
\hline Article Info & ABSTRACT \\
\hline Article history: & \multirow{10}{*}{$\begin{array}{l}\text { With increasing demand of air traffic, there is a need to optimize the use } \\
\text { of available airspace. Effective utilization of airspace relies on quality } \\
\text { of aircraft surveillance. Active research is carried out for enhancements in } \\
\text { surveillance techniques and various methods are evaluated for future use. } \\
\text { This paper evaluates the use of multiple signal classification (MUSIC) based } \\
\text { angle of arrival (AOA) estimation along with multiangulation for locating } \\
\text { aircrafts from their electromagnetic wave emission. The performance } \\
\text { evaluation of the system is presented by evaluating the AOA estimation errors } \\
\text { and position estimation (PE) errors. The errors are evaluated by comparing } \\
\text { the estimated value to the actual value. An analysis on the system parameters, } \\
\text { AOA error and PE error are presented in the end. AOA errors are affected by } \\
\text { the AOA value (emitter bearing), number of array elements, SNR and resolution } \\
\text { of AOA estimation algorithm. Errors in AOA estimation lead to PE errors. } \\
\text { The simulation results show small errors for short ranges. The system } \\
\text { performance can be improved at the expense of computational time by using } \\
\text { higher MUSIC resolution and larger antenna arrays. }\end{array}$} \\
\hline Received Dec 10, 2019 & \\
\hline Revised Feb 17, 2020 & \\
\hline Accepted May 4, 2020 & \\
\hline Keywords: & \\
\hline Angle of arrival estimation & \\
\hline Angulation & \\
\hline Position estimation & \\
\hline Position estimation error & \\
\hline Sensor array & \\
\hline
\end{tabular}

This is an open access article under the CC BY-SA license.

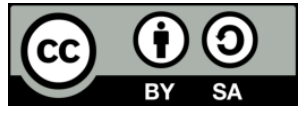

\section{Corresponding Author:}

Freeha Majeed Amjad,

School of Electrical Engineering,

Universiti Teknologi Malaysia,

81310, Skudai, Johor, Malaysia.

Email: fma1291@gmail.com

\section{INTRODUCTION}

Meeting the growing demand for air traffic largely depends on functionality of air traffic control (ATC) center which is responsible for locating aircraft accurately and updating each aircraft about the traffic in its surroundings [1]. Safety of air traffic flow depends on the quality of air traffic surveillance technique. Efficient surveillance can expedite the flow of air traffic and optimize the use of available airspace, hence allowing more aircrafts to fly simultaneously [2]. A typical air traffic control radar system consists of the primary surveillance radar (PSR) and secondary surveillance radar (SSR). The PSR estimates the target's position (bearing and range) by comparing the time difference between the transmitted and returned signal. With the use of SSR, the aircraft identity and altitude can be determined. The SSR sends an interrogation signal at an uplink frequency of $1030 \mathrm{MHz}$ while the aircraft's transponder replies at downlink frequency of $1090 \mathrm{MHz}$ [3]. There are 3 types of format used by SSR: mode A-identification, mode C-altitude and mode S-identification with altitude and position. Between the 3 , Mode $S$ is more advance to cater to the ever increase in air traffic intensity and to ensure navigation safety. Further enhancement comes with the introduction of ADS-B system which is a satellite based positioning system that utilizes GPS to estimate its current position (latitude and longitude) which is sent to the ground station along with other information such as velocity, altitude and 24 bits identification code. Aircrafts equipped with ADS-B transponders send signals periodically at 0.5 or 1 seconds at $1090 \mathrm{MHz}$ using one of the formats used in mode $\mathrm{S}$ namely the mode S Extended 
Squitter [4]. Thus, aircrafts are tracked at a more frequent rate compared to SSR at about 4 to 12 seconds which depends on the scan rate of the antenna [5]. Another technology to complement existing radar system is multilateration (MLAT) [6]. It estimates aircraft position by calculating the time difference of the aircraft transponder reply (mode $\mathrm{A}, \mathrm{C}$ and $\mathrm{S}$ ) detected at two spatial placed ground receiving stations (GRS)s. It requires a minimum of four GRSs which are all synchronized and connected to a command center via link infrastructure [7]. The ADS-B and SSR transponder transmit power for small aircraft is $125 \mathrm{~W}$ while for larger aircraft that flies at above 15,000 feet is $250 \mathrm{~W}$ [8]. Airborne weather radar is used by aircrafts to navigate and detect cloud formations. Since the radar operates in the X-band (8-12), it is possible to estimate the size and concentration of the atmospheric precipitation by measuring the energy difference of transmitted and returned signal [9]. The shorter wavelength allows the resolving smaller particles compared to the S-band [10]. The transmit power is lower at 500 Watts because of the shorter detection range and on board power constraint.

Search for both newer surveillance techniques and enhancements in the existing techniques is continuously going on. Although, Radar has been the conventional method for locating aircrafts, it requires high transmit power and high installation and maintenance cost, making it a costly surveillance technology. Furthermore, performance limitation due to distance, terrain and atmospheric condition and low update rate makes its functionality insufficient for surveillance in the current time [11]. Currently ADS-B alone suffers from security vulnerabilities due to its unauthenticated and unencrypted signals [12]. There are also chances of GPS device malfunction that would result in incorrect location [13]. Thus, there is a need to confirm the information provided by ADS-B system. In order to do so, time difference of arrival (TDOA) and angle of arrival (AOA) measurement based systems were suggested in [14]. An AOA based position estimation method is used as verification of ADS-B in [15]. A system based on MLAT is used to estimate 3D emitter position in [16]. A comparison of MLAT and multiangulation is carried out in [17]. Due to economic benefits of AOA, it is preferred over PSR, SSR, and MLAT.

In this paper, performance evaluation is carried out of an aircraft position estimation (PE) system based on a two stages process: 1) AOA estimation by multiple signal classification (MUSIC) algorithm, and 2) PE by angulation algorithm. Errors in each stage are analyzed. As for all wireless systems, noise is a major problem in aircraft surveillance. The received signals are corrupted by noise that leads to error in PE. If the maximum possible error in a system under a given operational condition (system coverage) is known, then this can be used to define a relationship between received signal quality and system performance. This relation can be used to identify the regions where the system will show promising results and where the system will fail. The errors in AOA estimation are evaluated by comparison of estimated AOA and actual AOA. The effect on the AOA errors is observed by variation in SNR values, number of array elements in receiver antenna and the resolution of MUSIC algorithm. Also, the effect on the computational time is examined. The AOA errors lead to PE errors in the second stage of system. A 2-D PE performance analysis of the system is presented to identify the range for which the system performs well.

\section{RESEARCH METHOD}

The system configuration is adopted from the previous works in [17, 18]. The system consists of four GRSs each equipped with a uniform linear array (ULA) all connected to the central unit as shown in Figure 1. The position coordinates of each GRS with a separation distance of $10 \mathrm{~km}$ are shown in Figure 2.

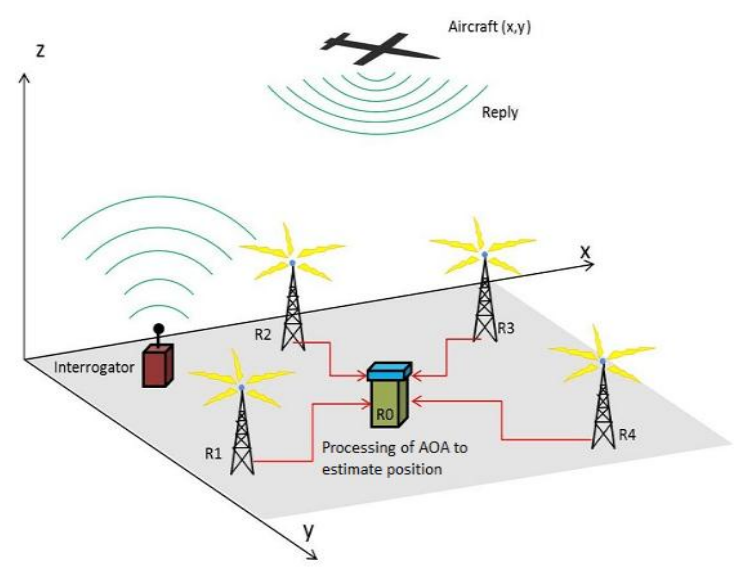

Figure 1. Working mechanism of AOA with maltiangulation 


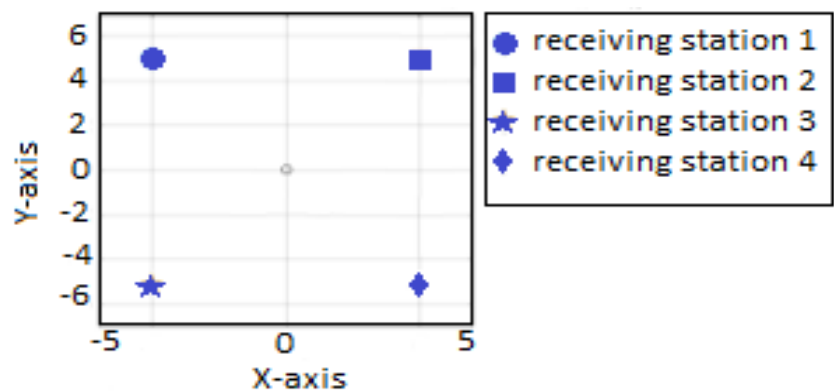

Figure 2. Receiving station configuration for 4 GRS

Simulation parameters used are based on true values used for aircraft surveillance. Signal with frequency of $1090 \mathrm{MHz}$, transmit power of $250 \mathrm{~W}$ and transmit antenna gain of $3 \mathrm{dBi}$ is used to represent the SSR and ADS-B transponder signal. Receiver antenna gain of $18 \mathrm{dBi}$ and $24 \mathrm{dBi}$ is used for 8 and 16 element arrays respectively. GRS receiver sensitivity is assumed to be $-90 \mathrm{dBm}$. PE errors are calculated for emitter range 5 to $200 \mathrm{~km}$ and emitter bearing $0^{\circ}$ to $359^{\circ}$. Signals transmitted by aircraft's transponder are received by each GRS simultaneously. Two variations of the MUSIC algorithm were used; one with $0.15^{\circ}$ resolution and the other with $0.25^{\circ}$ resolution. Root mean square error (RMSE) of position is used for comparison of PE error at various emitter positions. The PE process of the system is in two stages: 1) AOA estimation, and 2) PE using angulation algorithm. AOA estimation is carried out at each GRS while PE is done at the central unit. Firstly, signal received by each GRS is used for AOA estimation. In this system four AOAs are estimated for four GRSs. Next, the estimated AOAs along with the GRS position coordinates are used by the angulation algorithm to estimate the position of emitter.

\subsection{MUSIC algorithm for AOA estimation}

Several AOA estimation algorithms are present in the literature. These can be classified into subspace methods and non-subspace methods [19]. Non-subspace estimation techniques like beam forming and Capon's minimum variance method yield poor resolution and can identify only a single source at a time. Therefore, these algorithms are not suitable for air traffic monitoring. Subspace estimation techniques like MUSIC and estimation of signal parameter via rotational invariance technique (ESPRIT) have high resolution and perform efficiently where angles are indicated to be closely spaced [20]. These techniques can identify multiple sources given that the number of sensor elements in the sensor array is more than the number of emitters. A comparison shows that ESPRIT requires less computation than MUSIC and has a higher resolution. MUSIC on the other hand performs better at low SNR [21]. ESPRIT can show better accuracy but it requires twice as many sensor elements as MUSIC [22].

The ULA at each GRS consists of $M$ elements separated by a distance $d$. It receives the signal $s(t)$ from the emitter impinging the array at an angle $\theta$. The array element closest to emitter receives the signal earlier then the other elements. As a result, the other array elements receive a delayed version of $s(t)$. The signal at the $i$-th element is represented as:

$$
x_{i}(t)=e^{-j \omega(i-1)\left(\frac{d}{c}\right) \sin (\theta)} s(t)
$$

where $\omega=2 \pi f$ is the angular frequency, and $c$ is the speed of electromagnetic waves. Putting all the signals received at each GRS together with noise forms the input signal matrix $\mathrm{X}(t)$;

$$
\mathrm{X}(t)=\left[\begin{array}{c}
x_{1}(t) \\
x_{2}(t) \\
x_{3}(t) \\
\cdot \\
\cdot \\
\cdot \\
x_{M}(t)
\end{array}\right]+\mathbf{n}(t)=\left[\begin{array}{c}
1 \\
e^{-j \omega\left(\frac{d}{c}\right) \sin (\theta)} \\
e^{-j \omega\left(\frac{d}{c}\right) \sin (\theta)} \\
\cdot \\
\cdot \\
\cdot \\
e^{-j \omega(M-1) \frac{d}{c} \sin (\theta)}
\end{array}\right] s(t)+\mathbf{n}(t)
$$

$\mathrm{X}(t)=\mathrm{a}(\theta) s(t)+\mathrm{n}(t)$ 
where a $(\theta)$ is the steering vector that steers the received signal in the direction of the AOA [23]. Input signal matrix for $K$ emitters is given as:

$$
\mathrm{X}(t)=\mathrm{A}(\theta) s(t)+\mathrm{n}(t)
$$

where $\mathrm{A}(\theta)=\left[\mathrm{a}\left(\theta_{1}\right), \mathrm{a}\left(\theta_{2}\right), \mathrm{a}\left(\theta_{3}\right), \ldots \ldots, \mathrm{a}\left(\theta_{K}\right)\right]$. The MUSIC algorithm computes the $M \times M$ correlation matrix $\mathrm{R}_{\mathrm{x}}$.

$$
\mathrm{R}_{\mathrm{x}}=E\left\{\mathbf{x}(t) \mathbf{x}^{\mathrm{H}}(t)\right\}
$$

Eigenvalue decomposition of $\mathrm{R}_{\mathrm{x}}$ results in $M$ eigenvalues $\left(\lambda_{1}, \lambda_{2}, \lambda_{3}, \ldots, \lambda_{K}, \ldots, \lambda_{M},\right)$ and $M$ associated eigenvectors $\quad\left(\mathrm{u}_{1}, \mathrm{u}_{2}, \mathrm{u}_{3}, \ldots ., \mathrm{u}_{K}, \ldots, \mathrm{u}_{M}\right)$. Eigenvectors associated to $K$ smallest eigenvalues span the noise subspace $\mathrm{U}_{N}$ while the rest $(M-K)$ eigenvectors span the signal subspace $\mathrm{U} s$.

$$
\begin{aligned}
& \mathrm{U}_{N}=\left[\mathrm{u}_{1}, \mathrm{u}_{2}, \mathrm{u}_{3}, \ldots ., \mathrm{u}_{K}\right] \\
& \mathrm{U}_{S}=\left[\mathrm{u}_{K}, \mathrm{u}_{K+1}, \ldots . ., \mathrm{u}_{M}\right]
\end{aligned}
$$

Due to the orthogonality of the noise subspace and the array steering vector at the angles of arrival $\theta_{1}, \theta_{2}, \ldots$ $\theta_{K}$, the matrix product $\mathrm{a}^{H}(\theta) U_{N} U_{N}{ }^{H} \mathrm{a}(\theta)$ is zero for these angles. The reciprocal of this matrix product creates sharp peaks at the angle of arrival [24]. Thus, the MUSIC pseudospectrum is given by:

$$
P(\theta)=\frac{1}{\left|\mathbf{a}^{H}(\theta) \mathbf{U}_{N} \mathbf{U}_{N}{ }^{H} \mathbf{a}(\theta)\right|}
$$

\subsection{Multiangulation position estimation methodology}

Multiangulation is a method of estimating coordinates of emitter using AOAs and coordinates of GRS [8]. The point of intersection of line of bearings (LOB) is the position of emitter. Equation of LOB for the $\mathrm{i}$-th GRS is:

$$
y i=m i x i+c i
$$

where (xi,yi) are the coordinates of i-th GRS. The slope and the y-intercept for (9) are given as:

$$
\begin{aligned}
& m_{i}=\tan \left(90-\theta_{i}\right) \\
& c_{i}=y_{i}-x_{i} \tan \left(90-\theta_{i}\right)
\end{aligned}
$$

The multiangulation system consisting of four GRS results in four LOB equations which can be expressed in matrix form as:

$$
\mathrm{Ax}=\mathrm{b}
$$

where,

$$
\mathbf{A}=\left[\begin{array}{ll}
-\tan \left(90-\theta_{1}\right) & 1 \\
-\tan \left(90-\theta_{2}\right) & 1 \\
-\tan \left(90-\theta_{3}\right) & 1 \\
-\tan \left(90-\theta_{4}\right) & 1
\end{array}\right], \mathbf{x}=\left[\begin{array}{l}
x \\
y
\end{array}\right], \mathbf{b}=\left[\begin{array}{l}
y_{1}-x_{1} \tan \left(90-\theta_{1}\right) \\
y_{2}-x_{2} \tan \left(90-\theta_{2}\right) \\
y_{3}-x_{3} \tan \left(90-\theta_{3}\right) \\
y_{4}-x_{4} \tan \left(90-\theta_{4}\right)
\end{array}\right]
$$

Since the number of equations is more than the unknowns, the matrix inverse does not exist. The overdetermined least squares approach can be utilized [25]. Equation (12) can be represented as:

$$
\mathrm{A}^{T} \mathrm{~A} x=\mathrm{A}^{T} \mathrm{~b}
$$

The solution to the position estimate is found by:

$$
\mathrm{x}=\left(\mathrm{A}^{T} \mathrm{~A}\right)^{-1} \mathrm{~A}^{T} \mathrm{~b}
$$

For $N$-realization Monte Carlo simulation, RMSE for position is given by: 


$$
R M S E=\sqrt{\frac{\sum_{i=1}^{M}\left[\left(x_{i}-x\right)^{2}+\left(y_{i}-y\right)^{2}\right]}{N}}
$$

where $(x, y)$ are the actual position coordinates of emitter and $\left(x_{i}, y_{i}\right)$ are the position coordinates estimated at the $i$-th iteration.

\section{RESULTS AND DISCUSSION}

The evaluation of system performance is based on errors in AOA estimation and PE estimation.

\subsection{Effect of varying SNR on AOA estimation error}

The MUSIC algorithm was tested for various angles using Monte Carlo simulation for SNR values ranging from 25 to $55 \mathrm{~dB}$. Standard deviation of estimated angles is shown in Tables 1 and 2 for 8 and 16 elements array respectively. At low SNR AOA estimation degrades producing high unacceptable errors for AOA values closer to the edge of array shown by angles closer to $90^{\circ}$. Therefore, MUSIC algorithm will not be able to detect emitter at the edges of array at low SNR.

Table 1. AOA standard deviation for 8 elements array

Table 2. AOA standard deviation for 16

\begin{tabular}{lllllllll}
\hline $\mathrm{M}=8$ & \multicolumn{3}{c}{ SNR (dB) } & & & & \\
& & $\mathbf{2 5}$ & 30 & 35 & 40 & 45 & 50 & 55 \\
\hline AOA & 88 & $\mathbf{3 5}$ & 0.40 & 0.21 & 0.13 & 0.05 & 0 & 0 \\
$\left(^{\circ}\right)$ & 89 & $\mathbf{8 4}$ & $\mathbf{7 0}$ & $\mathbf{7 0}$ & $\mathbf{3 1}$ & 0.15 & 0.07 & 0.02 \\
& 90 & $\mathbf{8 9}$ & $\mathbf{8 8}$ & $\mathbf{8 9}$ & $\mathbf{8 7}$ & $\mathbf{8 6}$ & $\mathbf{8 2}$ & $\mathbf{7 4}$ \\
\hline
\end{tabular}

\begin{tabular}{lllllllll}
\hline $\mathrm{M}=15$ & \multicolumn{9}{l}{ SNR (dB) } & & & & & & \\
& & 25 & 30 & 35 & 40 & 45 & 50 & 55 \\
\hline AOA & 88 & 0.02 & 0.14 & 0.07 & 0 & 0 & 0 & 0 \\
$\left(^{\circ}\right)$ & 89 & $\mathbf{6 6}$ & $\mathbf{3 8}$ & 0.16 & 0.07 & 0 & 0 & 0 \\
& 90 & $\mathbf{9 0}$ & $\mathbf{8 8}$ & $\mathbf{8 8}$ & $\mathbf{8 5}$ & $\mathbf{8 3}$ & $\mathbf{7 5}$ & $\mathbf{6 6}$ \\
\hline
\end{tabular}

\subsection{Effect of varying number of array elements on AOA estimation error}

Tables 1 and 2 showed the performance of MUSIC algorithm is affected by the number of elements in the array. The 8 elements array is not able to estimate AOA of $88^{\circ}$ below $25 \mathrm{~dB}$ and $89^{\circ}$ below $40 \mathrm{~dB}$. However, the 16 elements array is able to estimate $88^{\circ}$ at any SNR value in the given range but cannot estimate $89^{\circ}$ below $30 \mathrm{~dB}$. The results also show that both the arrays cannot estimate AOA of $90^{\circ}$ at any SNR value. Therefore, at low SNR, increasing the number of array elements will improve the system performance

\subsection{Effect of varying MUSIC resolution on AOA estimation error}

MUSIC algorithm scans the spectrum for a range of $-90^{\circ}$ to $90^{\circ}$ with fixed interval defined by $t$ he resolution of algorithm. Smaller the interval higher is the resolution. For instance MUSIC with resolution of $1^{\circ}$ scans the spectrum at $-90^{\circ},-89^{\circ},-88^{\circ}, \ldots \ldots, 89^{\circ}, 90^{\circ}$ whereas MUSIC with a resolution of $5^{\circ}$ scans the spectrum at $-90^{\circ},-85^{\circ},-80^{\circ}, \ldots \ldots, 85^{\circ}, 90^{\circ}$. Resolution of MUSIC algorithm affects the performance of the system. A comparison of AOA RMSE for $0.15^{\circ}$ and $0.25^{\circ}$ resolution at angles between $27^{\circ}$ to $28^{\circ}$ is shown in Figure 3.

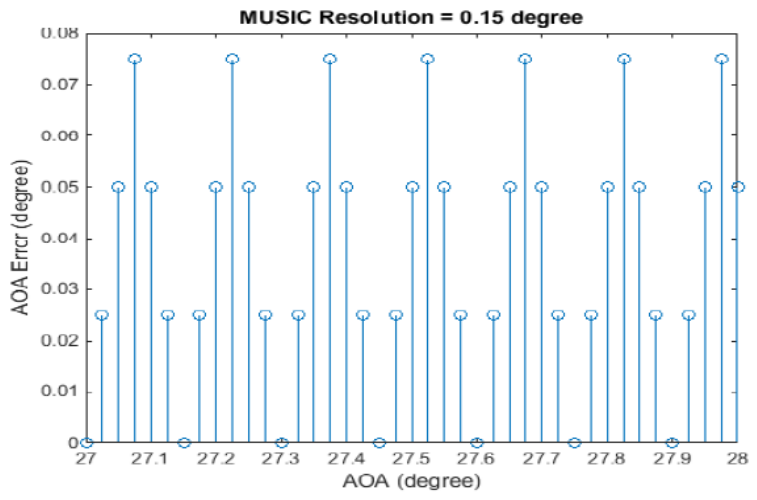

(a)

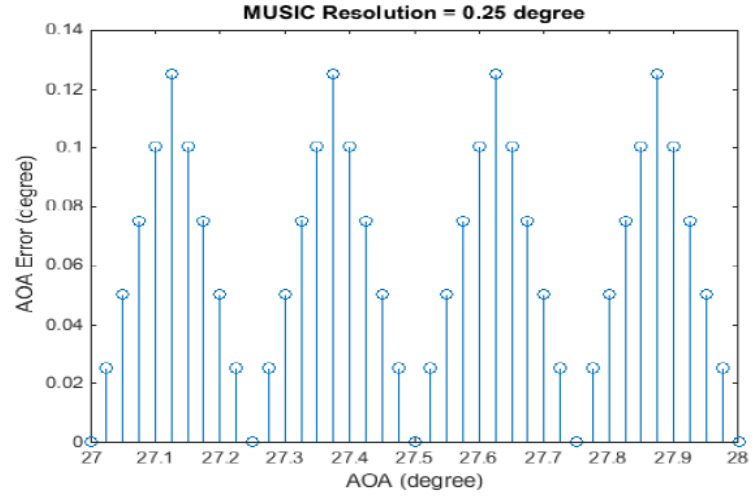

(b)

Figure 3. RMSE comparison for AOA estimation error, (a) AOA error for $0.15^{\circ}$ resolution, (b) AOA error for $0.25^{\circ}$ resolution 
From Figure 3 it can be seen that the error is zero for AOAs that are multiples of the resolution value such as at $27^{\circ}, 27.15^{\circ}, 27.30^{\circ}$ and $27.45^{\circ}$ for $0.15^{\circ}$ and at $27^{\circ}, 27.25^{\circ}, 27.50^{\circ}$ and $27.75^{\circ}$ for $0.25^{\circ}$. Amount of error at angles between the multiples of resolution value shows a symmetrical and periodic trend. Angles those are closer to the multiple of resolution value show low error.

\subsection{Effect of varying array elements on PE estimation error}

From the estimated AOA and the coordinates of all the four GRSs, the multiangulation algorithm is used to estimate the position of the emitter. The PE errors for various emitter positions are listed in Tables 3 and 4. The PE error that is less than $1.5 \mathrm{~km}$ satisfies Federal Aviation Administration (FAA) horizontal separation [16]. Both 8 and 16 element arrays show compliance with FAA horizontal separation for a range of $150 \mathrm{~km}$. Certain positions show high unacceptable errors and emitter at these positions cannot be detected by the multiangulation system with single ULA. At $0^{\circ}$ bearing and $5 \mathrm{~km}$ range, the actual AOA of the emitter at GRS-1 and GRS-2 are $90^{\circ}$ and $-90^{\circ}$ respectively. Since the actual AOA of $90^{\circ}$ cannot be estimated by MUSIC as shown in Table 1, a high PE error occurs at this position as shown in Tables 3 and 4 and the emitter cannot be detected by both 8 and 16 element arrays. It can be seen in Table 3 that high PE errors also occur at $90^{\circ}$ bearing for range beyond $150 \mathrm{~km}$ when using 8 elements array. The actual AOA at each GRS for these locations are above $88^{\circ}$ and SNR values are below $28 \mathrm{~dB}$. Table 1 shows high AOA errors for AOA above $88^{\circ}$ at SNR below $40 \mathrm{~dB}$. High AOA errors consecutively result in high PE errors.

\subsection{Effect of varying MUSIC elements on PE estimation error}

Figure 4(a)-(b) shows contour plot of PE errors for 16 array elements for $0.15^{\circ}$ and $0.25^{\circ}$ resolution respectively. The plots show that errors for $0.15^{\circ}$ resolution are lower than errors for $0.25^{\circ}$. This proves that MUSIC with higher resolution performs better. The errors increase as the range increases. It can also be seen that in Figure 4(b) there are certain positions with very high PE errors surrounded by positions with low PE errors. This is the result of variations in AOA errors by MUSIC resolution as shown in Figure 3. For $30^{\circ}$ bearing PE error at $50 \mathrm{~km}$ is $0.0244 \mathrm{~km}$ and at $150 \mathrm{~km}$ is $0.3412 \mathrm{~km}$. The PE error at $100 \mathrm{~km}$ is expected to be between 0.0244 and $0.3412 \mathrm{~km}$. However, the error at $100 \mathrm{~km}$ is $1.8552 \mathrm{~km}$. This is because emitter makes an angle of $26.16^{\circ}$ and $27.42^{\circ}$ with GRS-4 at $100 \mathrm{~km}$ and $150 \mathrm{~km}$ respectively. The AOA error for $26.16^{\circ}$ is higher than that for $27.42^{\circ}$ using a resolution of $0.15^{\circ}$. Thus, PE error at $100 \mathrm{~km}$ is higher than that at $150 \mathrm{~km}$.

Table 3. AOA standard deviation for 8 elements array

\begin{tabular}{lllllll}
\hline $\mathrm{M}=8$ & & $\begin{array}{l}\text { Bearing } \\
0^{\circ}\end{array}$ & $30^{\circ}$ & $45^{\circ}$ & $60^{\circ}$ & $90^{\circ}$ \\
\hline Range & 5 & $13.3029^{*}$ & 0.0063 & 0.0026 & 0.0049 & 0 \\
$(\mathrm{~km})$ & 50 & 0.1479 & 0.0244 & 0.3152 & 0.0284 & 0.0528 \\
& 100 & 0.7152 & 0.8982 & 0.1315 & 0.4224 & 0.4814 \\
& 150 & $\mathbf{2 . 6 9 2 4}$ & 0.2563 & 0.0592 & 0.3731 & $107.4^{*}$ \\
& 200 & 0.8114 & 0.6037 & 0.6773 & 0.6881 & $193.5^{*}$ \\
\hline
\end{tabular}

Table 4. AOA standard deviation for 16 elements array

\begin{tabular}{|c|c|c|c|c|c|c|}
\hline \multirow[t]{2}{*}{$M=16$} & & \multicolumn{5}{|l|}{ Bearing } \\
\hline & & $0^{\circ}$ & $30^{\circ}$ & $45^{\circ}$ & $60^{\circ}$ & $90^{\circ}$ \\
\hline \multirow{5}{*}{$\begin{array}{l}\text { Range } \\
(\mathrm{km})\end{array}$} & 5 & $12.2129^{*}$ & 0.0063 & 0.0026 & 0.0049 & 0 \\
\hline & 50 & 0.1479 & 0.0244 & 0.3160 & 0.0284 & 0.0354 \\
\hline & 100 & 0.7152 & 1.8552 & 0.1315 & 1.6183 & 0.0344 \\
\hline & 150 & 2.9790 & 0.3422 & 0.0583 & 0.8611 & 0.6230 \\
\hline & 200 & 1.5548 & 0.7601 & 0.6376 & 0.4988 & 1.8014 \\
\hline
\end{tabular}

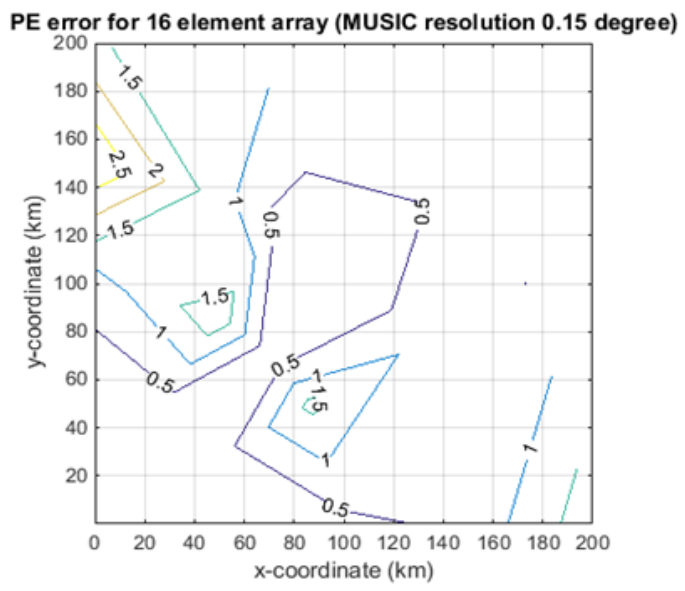

(a)

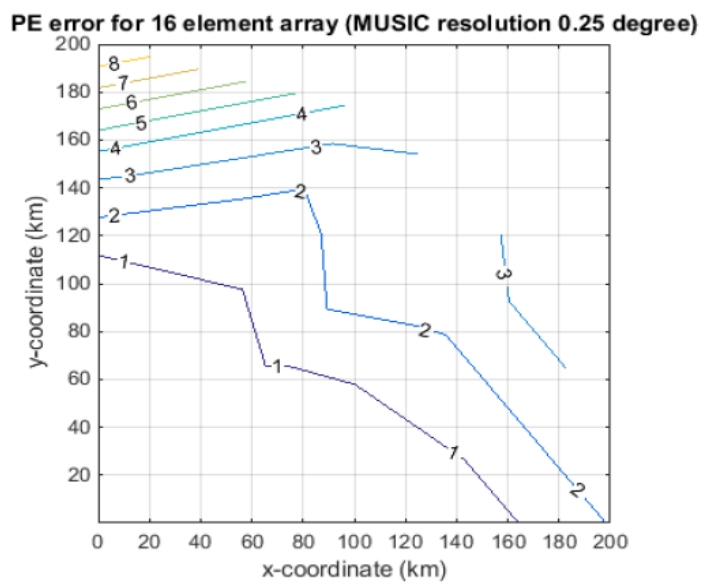

(b)

Figure 4. PE error comparison for MUSIC resolutions, (a) $0.15^{\circ}$ resolution, (b) $0.25^{\circ}$ resolution 


\section{CONCLUSION}

A performance evaluation of system for estimating the position of aircraft using the radar signals of aircraft transponder was presented. The system is based on AOA estimation combined with multiangultion algorithm to estimate the position. Errors in estimated AOA were observed by varying the number of elements in ULA, the SNR values of the received signal and the resolution of MUSIC algorithm. Lower number of array elements produces low receiver antenna gain. Thus, more AOA errors were observed using lesser number of array elements compared to higher number of array elements at a particular SNR. It was also observed that the angles closer to the edge of the array produce large AOA errors. This is due to the inability of MUSIC algorithm to detect AOA closer to the edge. A comparison was also made of the effect of resolution of MUSIC on AOA errors. A higher resolution produces lower AOA errors as compared to lower resolution. On the contrary it requires longer computational time. There is always a tradeoff between system performance and computational time based on MUSIC resolution. Errors in the estimated position depend on AOA errors and the range of emitter. Larger number of array elements and higher resolution of MUSIC algorithm result in lower PE errors. Position of aircraft closer to the system can be estimated with higher accuracy.

\section{ACKNOWLEDGEMENTS}

The authors would like to thank the authorities of Digital Signal Processing (DSP) lab, Universiti Teknologi Malaysia (UTM) for providing the tools to carry out this research.

\section{REFERENCES}

[1] M. S. Nolan, "Fundamentals of air traffic control," Delmar Cengage Learning, 2010.

[2] M. T. Treimuth, "Dynamic optimization of airspace sector grouping," Thesis, Mathématiques Appliquées, Université de Toulouse, Institut National Polytechnique de Toulouse, 2018.

[3] I. Cao, "Guidance material on comparison of surveillance technologies (GMST)," International Civil Aviation Organization, 2007.

[4] “ADS-B for dummies," European Organization for the Safety of Air Navigation.

[5] "ADS-B implementation and operations guidance document," Edition 11.0, International Civil Aviation Organization Asia and Pacific Office, 2018

[6] SRA International, "Multilateration \& ADS-B-Executive reference guide," Creativerge, 2009.

[7] F. A. Niles, R. S. Conker, M. B. El-Arini, D. G. O'Laighlin, and D. V. Baraban, "Wide area multilateration for alternate position, navigation, and timing (APNT)," MITRE-CAASD, Tech. Rep., (2012).

[8] D. W. Hempe, "Airworthiness approval of automatic dependent surveillance-broadcast (ADS-B) out systems," Advisory Circular 20-165B, pp. 1-113, 2010.

[9] Avition Knowledge, "Interpretation of weather radar," 2011. [Online]. Available at: http://aviationknowledge.wikidot.com/aviation:interpretation-of-weather-radar.[Accessed 78 2019].

[10] Weather Edge Inc., "Weather radar, an online guide for the remote sensing of precipitation," 2000. [Online]. Available at: http://www.everythingweather.com/weather-radar/bands.shtml. [Accessed 38 2019].

[11] A. Abdulaziz, A. S. Yaro, A. A. Ahmad, and S. Namadi, "Surveillance radar system limitations and the advent of the automatic dependent surveillance broadcast system for aircraft monitoring," Journal of Science, Technology \& Education (JOSTE), vol. 7, no. 2, pp. 9-15, 2019.

[12] M. Strohmeier, V. Lenders, and I. Martinovic, "On the security of the automatic dependent surveillance-broadcast protocol," IEEE Communications Surveys \& Tutorials, vol. 17, no. 2, pp. 1066-1087, 2015.

[13] L. Purton, H. Abbass, and S. Alam, "Identification of ADS-B system vulnerabilities and threats," Australasian Transport Research Forum 2010 Proceedings, Canberra, pp. 1-16, 2010.

[14] Y. A. Nijsure, G. Kaddoum, G. Gagnon, F. Gagnon, C. Yuen, and R. Mahapatra, "Adaptive air-to-ground secure communication system based on ADS-B and wide-area multilateration," IEEE Transactions on Vehicular Technology, vol. 65, no. 5, pp. 3150-3165, May 2016.

[15] C. Reck, M. S. Reuther, A. Jasch, and L-P. Schmidt, "Verification of ADS-B positioning by direction of arrival estimation," International Journal of Microwave and Wireless Technologies, vol. 4, no. 2, pp. 181-186, 2012.

[16] A. Z. Sha'ameri, Y. A. Shehu, and W. Asuti, "Performance analysis of a minimum configuration multilateration system for airborne emitter position estimation," Defence S and T Technical Bulletin, vol. 8, no. 1, pp. 27-41, 2015.

[17] A. Z. Sha'ameri, A. S. Yaro, F. M. Amjad, and M. N. M. Hamdi, "Performance comparison of emitter locating system for low level airborne targets," Defence S and T Technical Bulletin, vol. 10, no. 3, pp. 199-217, 2018.

[18] F. M. Amjad, A. Z. Sha'ameri, A. S. Yaro, and S. M. Yarima, "Position estimation error for multiangulation system for air traffic monitoring," Symposium on Acoustic, Speech and Signal Processing (SASSP), 2019.

[19] R. A. Poisel, "Electronic warfare target location methods," Norwood: Artech House, 2012.

[20] D. K. Mondal, B. Dian, C. Dutta, A. Chaterjee, and A. Ray, "A brief study of the ESPRIT direction of arrival estimation algorithm in an uncorrelated environment for application in the smart antenna," Communication and Electrical Engineering: Proceedings of the 3rd International Conference on Foundations and Frontiers in Computer, Communication and Electrical Engineering, (C2E2-2016). Taylor \& Francis Books, pp. 541-546, 2016. 
[21] G. P. Chowdary, "Performance comparison of various DOA estimation techniques based on antenna parameter," International Journal of Review in Electronics and Communication Engineering, vol. 4, no. 2, pp. 15-19, 2016.

[22] N. Waweru, D. Konditi, and P. Langat, "Performance analysis of MUSIC, root-MUSIC and ESPRIT DOA estimation algorithm," International Journal of Electronics and Communication Engineering, vol. 8, no. 1, pp. 209-216, 2014.

[23] H. H. Fan, "Direction of arrival estimation (DOA) in interference \& multipath propagation," [Online]. Available at: https://www.girdsystems.com/wordpress/wp-content/uploads/2019/05/GIRD_Systems_DOA_Multipath.pdf. [Accessed 38 2019].

[24] H. Tang, "DOA estimation based on MUSIC algorithm," Thesis, Department of Physics and Electrical Engineering, Linnaeus University, 2014.

[25] H. Anton and C. Rorres, "Elementary linear algebra," John Wiley \& Sons, 2010.

\section{BIOGRAPHIES OF AUTHORS}

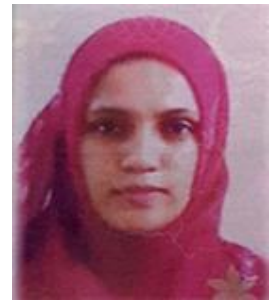

Freeha Majeed Amajd received BS degree in Electrical Engineering from National University of Computer and Emerging Sciences, Islamabad, Pakistan and MS degree in Electrical Engineering from Universiti Teknologi Malaysia (UTM), Skudai. She is currently pursuing Ph.D. degree at School of Electrical Engineering, Universiti Tecknologi Malaysia, Skudai. Her research interests include signal processing for communication, localization and position estimation and internet of things.

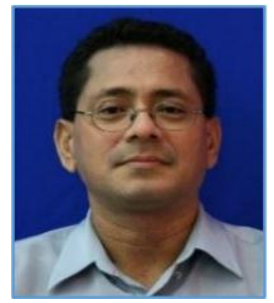

Ahmad Zuri Sha'ameri obtained his B. Sc. in Electrical Engineering from the University of Missouri-Columbia, USA in 1984, and M. Eng. Electrical Engineering and PhD both from UTM in 1991 and 2000 respectively. At present, he is an associate professor, Coordinator for the Digital Signal and Image Processing (DSIP) Research Group and Academic Coordinator for the DSP Lab, Electronic and Computer Engineering Department, Faculty of Electrical Engineering, UTM. His research interest includes signal theory, signal processing for radar and communication, signal analysis and classification, and information security. The subjects taught at both undergraduate and postgraduate levels include digital signal processing, advance digital signal processing, advance digital communications and information security. He has also conducted short courses for both government and private sectors. At present, he has published 160 papers in his areas of interest at both national and international levels in conferences and journals.

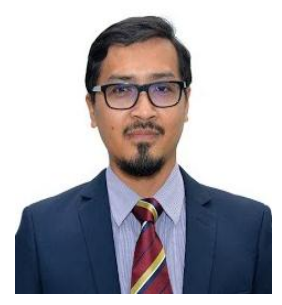

Kamaludin Mohamad Yusof received the B. Eng degree in Electrical - Electronics Engineering and M. Eng degree in Electrical Engineering from Universiti Teknologi Malaysia. He received $\mathrm{Ph}$. D degree from University of Essex, U.K. He is currently a senior lecturer in the Department of Communication Engineering, School of Electrical Engineering, Faculty of Engineering and member of Communication \& Network System (CNeTS) at Universiti Teknologi Malaysia. His current research interests include Internet-of-Things, Big Data and Software-Defined Network.

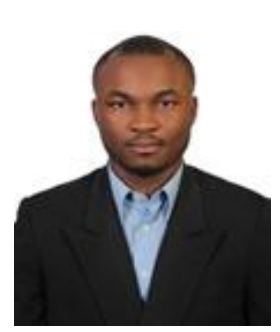

Paulson Eberechukwu Numan received his Bachelors in Engineering degree (Electrical and Electronics) in 2012 from the Federal University of Technology Akure, Ondo state, (Nigeria's topmost University of Technology). He obtained his Master of Engineering degree (Telecommunications and Electronics) in 2017 with a distinction and a recipient of the Best Post Graduate Student Award (BPGSA) from the prestigious Universiti Teknologi Malaysia (UTM), Johor, Malaysia. He is currently pursuing the Doctor of Philosophy Degree with the Faculty of Electrical Engineering, UTM. His general research interest lies in the field of wireless communications, computer networking system design and optimization. In particular, he is interested in Software Defined Networks (SDN), Internet of Things (IoT), Cognitive Radio Networks (CRN), and Wireless Sensor Networks (WSN). 